\title{
Lysines 207 and 325 methylation of WDR5 catalyzed by SETD6 promotes breast cancer cell proliferation and migration
}

\author{
RUOSI YAO ${ }^{1,2}$, YINGLI WANG ${ }^{3}$, DANYANG HAN ${ }^{2}$, YUHUI MA ${ }^{1}$, MUSONG MA $^{4}$, \\ YAPING ZHAO ${ }^{1}$, JIANG TAN ${ }^{1}$, JUN LU ${ }^{1}$, GUIYING XU ${ }^{4}$ and XIAOXUE LI ${ }^{1}$ \\ ${ }^{1}$ Key Laboratory of Molecular Epigenetics of the Ministry of Education, Northeast Normal University, \\ Changchun, Jilin 130024; ${ }^{2}$ Department of Hematology, Blood Diseases Institute, Xuzhou Medical University, \\ Xuzhou, Jiangsu 221004; ${ }^{3}$ Department of Toxicology, Jilin Province Institution of Tumor Research; \\ ${ }^{4}$ Department of Breast Surgery, Tumor Hospital of Jilin Province, Changchun, Jilin 130000, P.R. China
}

Received November 13, 2017; Accepted August 14, 2018

DOI: 10.3892/or.2018.6669

\begin{abstract}
Accumulating evidence has revealed that the methylation of lysines on nonhistones by histone lysine methyltransferases (HMTs) is crucial for regulating tumorigenesis and metastasis. However, whether the methylation of lysines on HMT complex components occurs and has functions in cancer progression is less well understood. WD repeat domain 5 (WDR5) is a core component of an HMT complex named mixed lineage leukemia (MLL)/Suppressor of Variegation, Enhancer of Zeste, and Trithorax 1 (SET1). In the present study, it was reported that lysines 207 and 325 (K207 and K325, respectively) of WDR5 were monomethylated by SET-domain-containing protein methyltransferase 6 . Disrupting the methylation of K207/K325 via a K207R/K325R double-site mutation attenuated the WDR5 promotion of breast cancer cell proliferation and migration. Methylation of K207/K325 on WDR 5 partially contributed to maintaining global histone tri-methylation of lysine 4 on histone H3 levels, but did not affect MLL/SET1 complex assembly. These results further understanding of a potential post-translational modification of WDR5, and imply that the methylation of lysines on HMT complex components is crucial for regulating human carcinogenesis.
\end{abstract}

Correspondence to: Dr Xiaoxue Li, Key Laboratory of Molecular Epigenetics of the Ministry of Education, Northeast Normal University, 5268 Renmin Street, Changchun, Jilin 130024, P.R. China E-mail: lixx956@nenu.edu.cn

Dr Guiying $\mathrm{Xu}$, Department of Breast Surgery, Tumor Hospital of Jilin Province, 1018 Huguang Road, Changchun, Jilin 130000, P.R. China

E-mail: 2831807050@qq.com

Key words: WD repeat domain 5, lysine methylation, SET domain containing protein methyltransferase 6 , breast cancer, cell proliferation, cell migration

\section{Introduction}

WD repeat domain 5 (WDR5) is a core component of histone lysine methyltransferase (HMT) complex, mixed lineage leukaemia (MLL)/Suppressor of Variegation, Enhancer of Zeste, and Trithorax 1 (SET1) (1). Through binding tri-methylation of lysine 4 on histone $\mathrm{H} 3$ (H3K4me3) methyltransferase MLL1 to a K4-dimethylated H3 tail, WDR5 is crucial for controlling H3K4me3 expression (2). Previous studies have revealed that WDR5 has key functions in regulating embryonic stem cell self-renewal (3) and vertebrate development (4). WDR5 is highly expressed in prostate, bladder and colorectal cancer, and acute leukemia; and high levels of WDR5 are positively correlated with an advanced cancer stage and poor survival rates (5-8). Knockdown of WDR5 in leukemia and bladder cancer cells is reported to suppress cell proliferation $(8,9)$. These results imply that WDR5 is likely to function in cancer progression.

Methylation of lysines or arginines on proteins is a classical and conserved protein post-translational modification catalyzed by HMTs and protein arginine methyltransferases (PRMTs) (10). In addition to histone methylation, evidence has revealed that the methylation of non-histone functions in physiological and pathological events, including in carcinogenesis and metastasis $(11,12)$. Tumor repressor p53 is reported to be monomethylated at the three lysine residues 370,372 and 382 within the regulatory C-terminal region by lysine methyltransferases SET domain containing lysine methyltransferase 7 (SET7/9), SET and MYND Domain Containing 2 and SET8, resulting in changes to p53 activity (13-15). SET-domaincontaining protein methyltransferase6 (SETD6), a HMT, methylates p21-activated kinase 4 (PAK4) to enhance the interaction of PAK4 with $\beta$-catenin and promote the $\mathrm{Wnt} / \beta$-catenin pathway (16).

In addition to lysine methylation, growing evidence has demonstrated the effect of arginine methylation on non-histones in controlling cancer progression (17). Methylation of arginine 1064 on BRG1-associated factor 155, which is catalyzed by a PRMT, co-activator-associated methyltransferase 1 , regulates breast cancer cell migration and metastasis (18). Protein arginine methyltransferase 5 methylates 
p53 at arginines 333, 335 and 337, increasing the activity of p53 in response to DNA repair (19). Previous evidence reveals that certain PRMTs, including PRMT7, are automethylated in breast cancer cells (20). However, whether the components of HMT complexes are methylated remains less well investigated.

In the present study, the results shed light on a novel post-translational modification of WDR5 and imply that the methylation of components of histone methyltransferase complexes is critical for regulating human carcinogenesis and cancer progression.

\section{Materials and methods}

Cell culture. MCF10A, MCF7, MDA-MB-231, BT549 and $293 \mathrm{~T}$ cell lines were purchased from the American Type Culture Collection (Manassas, VA, USA). MCF10A cells were cultured as previously described (21). MCF7 and BT549 cells were cultured in RPMI-1640 medium (Sigma-Aldrich; Merck KGaA, Darmstadt, Germany) supplemented with $10 \%$ fetal bovine serum (FBS; Shanghai ExCell Biology, Inc., Xuhui, Shanghai, China) and $0.023 \mathrm{IU} / \mathrm{ml}$ insulin at $37^{\circ} \mathrm{C}$ with $5 \% \mathrm{CO}_{2}$. MDA-MB-231 cells were cultured in L15 medium (Sigma-Aldrich; Merck KGaA) with 10\% FBS at $37^{\circ} \mathrm{C}$ without $\mathrm{CO}_{2} .293$ cells were cultured in DMEM (Sigma-Aldrich; Merck KGaA) containing $10 \% \mathrm{FBS}$ at $37^{\circ} \mathrm{C}$ with $5 \% \mathrm{CO}_{2}$.

Antibodies and plasmids. The following antibodies were used in western blot assays: Anti-WDR5 (cat. no. 07-706; EMD Millipore, Billerica, MA, USA), anti-histone H3 (cat. no. 06-755; EMD Millipore), anti-H3K4me3 (cat. no. 07-473; EMD Millipore), anti-methylated Lysine (cat. no. ab76118; Abcam, Cambridge, UK), anti-glutathione S-transferase (GST; cat. no. KM8005; SunGene GmbH, Gatersleben, Saxony-Anhalt, Germany) and anti- $\beta$-actin (cat. no. A5441; Sigma-Aldrich; Merck KGaA). Anti-His (cat. no. KM8001; SunGene GmbH) and anti-Flag (cat. no. M20008M; Abmart, Inc., Berkley Heights, NJ, USA) were used for immunoprecipitation assays.

The cDNA of WDR5 was cloned into plasmid pET-28a, p3*Flag-cmv-10 and lentiviral expression plasmid Pwpxld (Addgene, Inc., Cambridge, MA, USA). Using QuikChange (Stratagene; Agilent Technologies, Inc., Santa Clara, CA, USA) according to the manufacturer's protocol, the asynonymous mutation of WDR5 (rWDR5) was generated, and lysines 207 and 325 of WDR5 were either mutated separately or together into arginines to generate K207R, K325R single-site mutation and K207R/K325R (2KR) double-site mutation. The p3*Flag-CMV-10 plasmids expressing wild-type (WT) WDR5, mutant (K207R, K325R or 2KR) WDR5 or rWDR5 were transfected into 293T cells using the PolyJet in vitro DNA transfection reagent (cat. no. SL100688; SignaGen Laboratories, Rockville, MD, USA) for $6 \mathrm{~h}$ at room temperature.

The cDNAs of SETD6, SET7/9, SET8 and SET and MYND Domain Containing 3 (SMYD3) were cloned into the pGEX-6P-1 plasmid (Addgene, Inc.). Using the aforementioned method, tyrosine 261 of SETD6 was mutated into alkaline (Y261A), and mutant (K207R, K325R or 2KR) WDR5 were generated in pGEX-6P-1 plasmids.
RNAi. Oligonucleotides short hairpin RNA against WDR5 (shWDR5)\#1 and shWDR5\#2 were designed and cloned into the pDSL-hpUGIP vector (Addgene, Inc.). shCtrl was used as the scrambled control shRNA. These lentiviral plasmids (pDSL-hpUGIP-shCtrl, pDSL-hpUGIP-shWDR5\#1 and \#2) and corresponding packaging vectors (PAX and PMG2.G; Addgene, Inc.) were used for generating lentivirus in 293T cells at a density of $1.5 \times 10^{6} / \mathrm{ml}$ in $10 \mathrm{~cm}$ culture vessel using the PolyJet in vitro DNA transfection reagent (SignaGen Laboratories) for $6 \mathrm{~h}$ at room temperature. After $48 \mathrm{~h}$, the lentiviral particles were harvested and concentrated using $100 \mathrm{~K}$ centrifugal filters (EMD Millipore). The viral titer was determined by counting plaques according to the dilution of virus plated and the volume of virus solution placed on the single monolayer. Lentiviral particles were incubated with breast cancer cells for $24 \mathrm{~h}$ to silence WDR5 expression at room temperature. The sequences of the shRNAs primers used were as follows: shCtrl forward, 5'-GATCCCCTTCTC CGAACGTGTCACGTTTCAAGAGAACGTGACACGTTC GGAGAATTTTTC-3' and reverse, 5'-TCGAGAAAAA TTCTCCGAACGTGTCACGTTCTCTTGAAACGTGACAC GTTCGGAGAAGGG-3'; shWDR5\#1 forward, 5'-GATC CCCGCAAGTTCATCTGCTGATATTCAAGAGATATCAG CAGATGAACTTGCTTTTTC-3' and reverse, 5'-TCGAGA AAAAGCAAGTTCATCTGCTGATATCTCTTGAATATCA GCAGATGAACTTGCGGG-3'; shWDR5\#2 forward, 5'-GAT CCCCGAATGAGAAATACTGCATATTCAAGAGA TAT GCAGTATTTCTCATTCTTTTTC-3' and reverse, 5'-TCGA GAAAAAGAATGAGAAATACTGCATATCTCTTGAAT ATGCAGTATTTCTCATTCGGG-3'.

Expression and purification of proteins expressed in bacteria. Plasmids pET28a (Addgene, Inc.) and pGEX-6P-1 containing each target gene (SETD6, SET8, SET7/9 and SMYD3) were transformed into BL21 bacteria and induced with isopropyl $\beta$-D-1-thiogalactopyranoside (Sigma-Aldrich; Merck KGaA) for $6 \mathrm{~h}$ at $25^{\circ} \mathrm{C}$. Histidine-(His-) tagged proteins were purified by Ni-NTA resin (GE Healthcare, Chicago, IL, USA) as previously described (22). Imidazole (50 mM, $100 \mathrm{mM}$ and $250 \mathrm{mM}$; Sigma-Aldrich;Merck KGaA) was used for eluting the His-WDR5 proteins at room temperature for $30 \mathrm{~min}$. GST-tagged proteins were purified with glutathione Sepharose 4B beads (GST fusion proteins; GE Healthcare) as previously described (23).

In vitro methylation assay and mass spectrometry analysis. Purified His-WDR5 fusion proteins were incubated with whole-cell extracts of MDA-MB-231 cells and 2 mCi S-adenosyl-L-[methyl- $\left.{ }^{3} \mathrm{H}\right]$ methionine $\left({ }^{3} \mathrm{H}-\mathrm{SAM}\right.$; GE Healthcare), which was used as a methyl donor, in a mixture of $30 \mu \mathrm{l}$ methylation reaction buffer (GE Healthcare) for $1 \mathrm{~h}$ at $30^{\circ} \mathrm{C}$. The reaction was stopped by adding $5 \mathrm{X}$ loading buffer (Bio-Rad Laboratories, Inc., Hercules, CA, USA) followed by $10 \%$ SDS-PAGE, the gel was dried and autoradiographed at $-80^{\circ} \mathrm{C}$. The in vitro methylation assays were performed by utilizing unlabeled S-adenosyl-methionine (SAM) as a methyl donor. Followed by $10 \%$ SDS-PAGE, the proteins were stained by $0.25 \%$ Coomassie brilliant blue (CBB) for $10 \mathrm{~min}$ at room temperature. The methylated His-WDR5 proteins in the CBB stained band were excised for liquid chromatography-tandem mass spectrometry analysis (LC-MS/MS) performed at the 
Institute of Biophysics (Chinese Academy of Sciences, Beijing, China).

LC-MS/MS analysis was performed on an EASY-nLC 1200 liquid chromatographic system coupled to a Q-Exactive mass spectrometer (Thermo Fisher Scientific, Inc., Waltham, MA, USA). The mass spectrometer was equipped with a nano-electrospray ionization source operated in the positive ionization mode. An aliquot of $1 \mu \mathrm{g}$ peptides was injected and trapped on an Acclaim PepMap ${ }^{\mathrm{TM}} 100$ column $(100 \mu \mathrm{m} \times 2 \mathrm{~cm}$, $5 \mu \mathrm{m}, 100 \AA$ ), and then separated on an Acclaim PepMap ${ }^{\mathrm{TM}}$ rapid seperation liquid chromatography column $(50 \mu \mathrm{m} \times 15 \mathrm{~cm}$, $2 \mu \mathrm{m}, 100 \AA$; Thermo Fisher Scientific, Inc.). The linear gradient used to separate peptides was set as follows: $5-30 \%$ B over $80 \mathrm{~min}$; 30-40\% B over $16 \mathrm{~min}$ and 40-100\% B over $8 \mathrm{~min}$. The mobile phase A was $0.1 \%$ formic acid (FA), and the mobile phase $\mathrm{B}$ was $0.1 \% \mathrm{FA}$ in $80 \%$ acetonitrile. The flow rate was $300 \mathrm{nl} / \mathrm{min}$. The mass spectrometer was operated with following parameters: Spray voltage, $1.9 \mathrm{kV}$; capillary temperature, $300^{\circ} \mathrm{C}$; probe heater temperature, $350^{\circ} \mathrm{C}$ and S-lens RF level, 60\%. Data was acquired using data-dependent acquisition mode, which could automatically switch MS to MS/MS scans. The MS scans were acquired in the range of $\mathrm{m} / \mathrm{z} 350-2,000$ with a resolution of 70,000. The automatic gain control (AGC) target value was set at $3 \mathrm{e} 6$, and the maximum injection time was $50 \mathrm{~ms}$. The 15 most abundant precursors were considered to fragment using higher energy collisional dissociation with a normalized collision energy of $27 \%$. The resolution for MS/MS analysis was set as 17,500, with an AGC target value of 1e5. Dynamic exclusion of target ions was set as $60 \mathrm{~s}$. The system control and data acquisition were performed on Xcalibur software (version 2.1; Thermo Fisher Scientific, Inc.) and data analysis was performed on the Proteome Discoverer software (version 2.2; Thermo Fisher Scientific, Inc.).

Immunoprecipitation. This procedure was performed as previously described (21). For immunoprecipitation, the 293 T cells were lysed using RIPA lysis buffer (Beyotime Institute of Biotechnology, Haimen, China) at $4^{\circ} \mathrm{C}$ for $30 \mathrm{~min}$. The total whole-cell extracts were incubated at $4^{\circ} \mathrm{C}$ overnight with anti-WDR5 [1:1,000; rabbit immunoaffinity purified immunoglobulin G (IgG); cat. no. 07-706; EMD Millipore], and gently shaken at $4^{\circ} \mathrm{C}$, followed by the addition of $20 \mu \mathrm{l}$ protein A magnetic beads for another $2 \mathrm{~h}$. Then the beads were washed in Buffer A (cat. no. P0013; Beyotime Institute of Biotechnology) for 3 times. The beads were resuspended in $100 \mu \mathrm{l} 2 \mathrm{X}$ loading buffer and boiled $\left(100^{\circ} \mathrm{C}\right)$ for $10 \mathrm{~min}$, followed by centrifugation at $10,000 \mathrm{x}$ for $15 \mathrm{~min}$ at $4^{\circ} \mathrm{C}$. The experiments were replicated at least 3 times.

Western blotting. The proteins obtained from the above immunoprecipitation were first quantified using the BCA Protein Assay kit (Thermo Fisher Scientific, Inc.; cat. no. NCI3225CH), then $10 \mu \mathrm{g}$ protein per lane were separated by $10 \%$ SDS-PAGE, then transferred to a $0.45 \mu \mathrm{m}$ pore size polyvinylidene fluoride membrane (EMD Millipore; cat. no. IPVH00010). Subsequent to blocking with 5\% non-fat milk at room temperature for $1 \mathrm{~h}$, the membrane was labeled with primary antibodies (Flag, 1:1,000, cat. no. M20008M; Abmart, Inc.; Lysine-me, 1:2,000, cat. no. ab76118; Abcam) at $4^{\circ} \mathrm{C}$ overnight and secondary antibodies (horseradish peroxidase-conjugated rabbit IgG; 1:5,000;
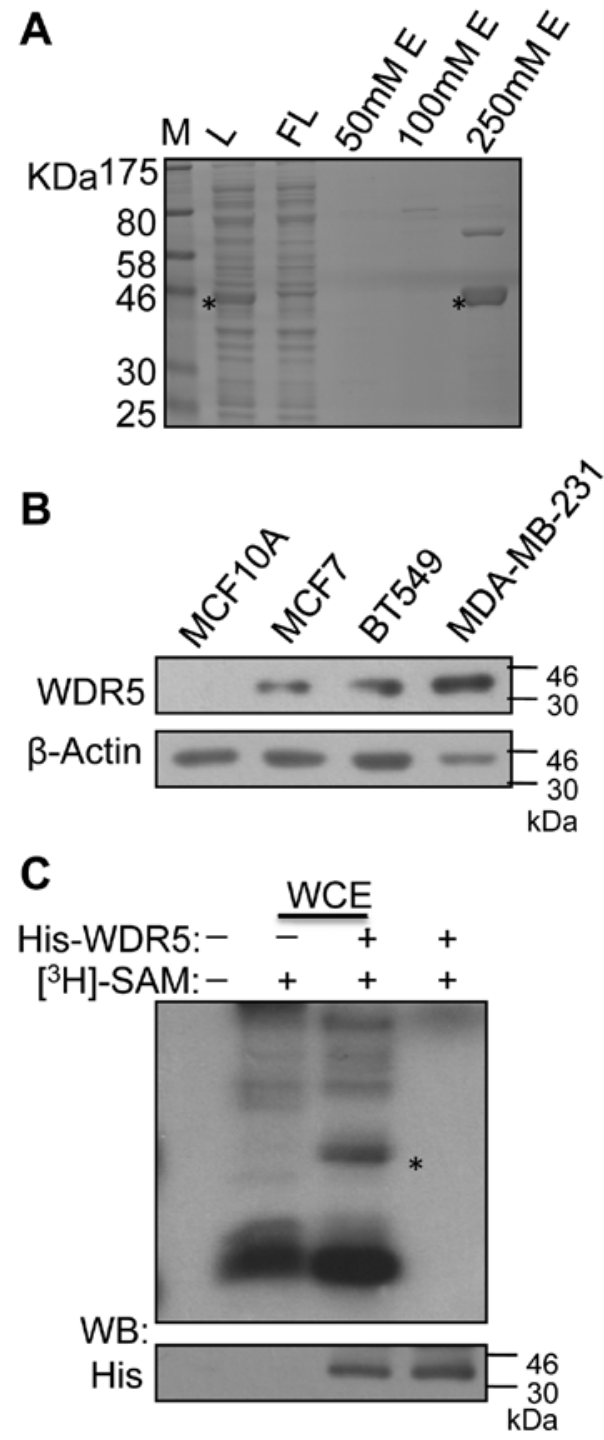

Figure 1. WDR5 was methylated by the WCE of breast cancer cells in vitro. (A) Wild-type His-WDR5 proteins expressed in bacteria were purified with Ni-NTA resin by using different concentrations of imidazole. Asterisks indicate the position of His-WDR5 proteins. $\mathrm{L}=$ lysate, $\mathrm{FL}=$ flow-through and E=elution. (B) WDR5 was highly expressed in MDA-MB-231 breast cancer cells. WDR5 expression level was detected by immunoblotting the WCE of each breast cancer cell line (MCF7, BT549 and MDA-MB-231) with anti-WDR5 antibody. Mammary epithelial MCF10A cells were used as negative controls. $\beta$-Actin was used as an internal reference.(C) His-WDR5 proteins were methylated in the WCE of MDA-MB-231 cells in vitro. Purified His-WDR5 proteins expressed in bacteria were incubated with or without the WCE of MDA-MB-231 cells for in vitro methylation reactions. His-WDR 5 protein levels were measured by WB analysis. $\left[{ }^{3} \mathrm{H}\right]-\mathrm{SAM}$ was used as a methyl donor. Methylation of His-WDR5 proteins was revealed by autoradiography and marked by an asterisk. WDR5, WD repeat domain 5; His-, histidine-tagged; WCE, whole-cell extract; $\left[{ }^{3} \mathrm{H}\right]-\mathrm{SAM}$, S-adenosyl-L-[methyl- $\left.{ }^{3} \mathrm{H}\right]$ methionine; WB, western blotting.

Proteintech Group, Inc., Chicago, IL, USA) at room temperature for $1.5 \mathrm{~h}$. The protein bands were detected by electrochemiluminesence substrate (Bio-Rad Laboratories, Inc.) and visualized by Imagequant LAS 4000 (GE Healthcare). The densitometric analysis of the bands were conducted by Image J 1.46 software (National Institutes of Health, Bethesda, MD, USA).

Colony formation assay. The MCF7 cells were harvested and seeded into 6-well plates (1,000 cells/well) and cultured at 

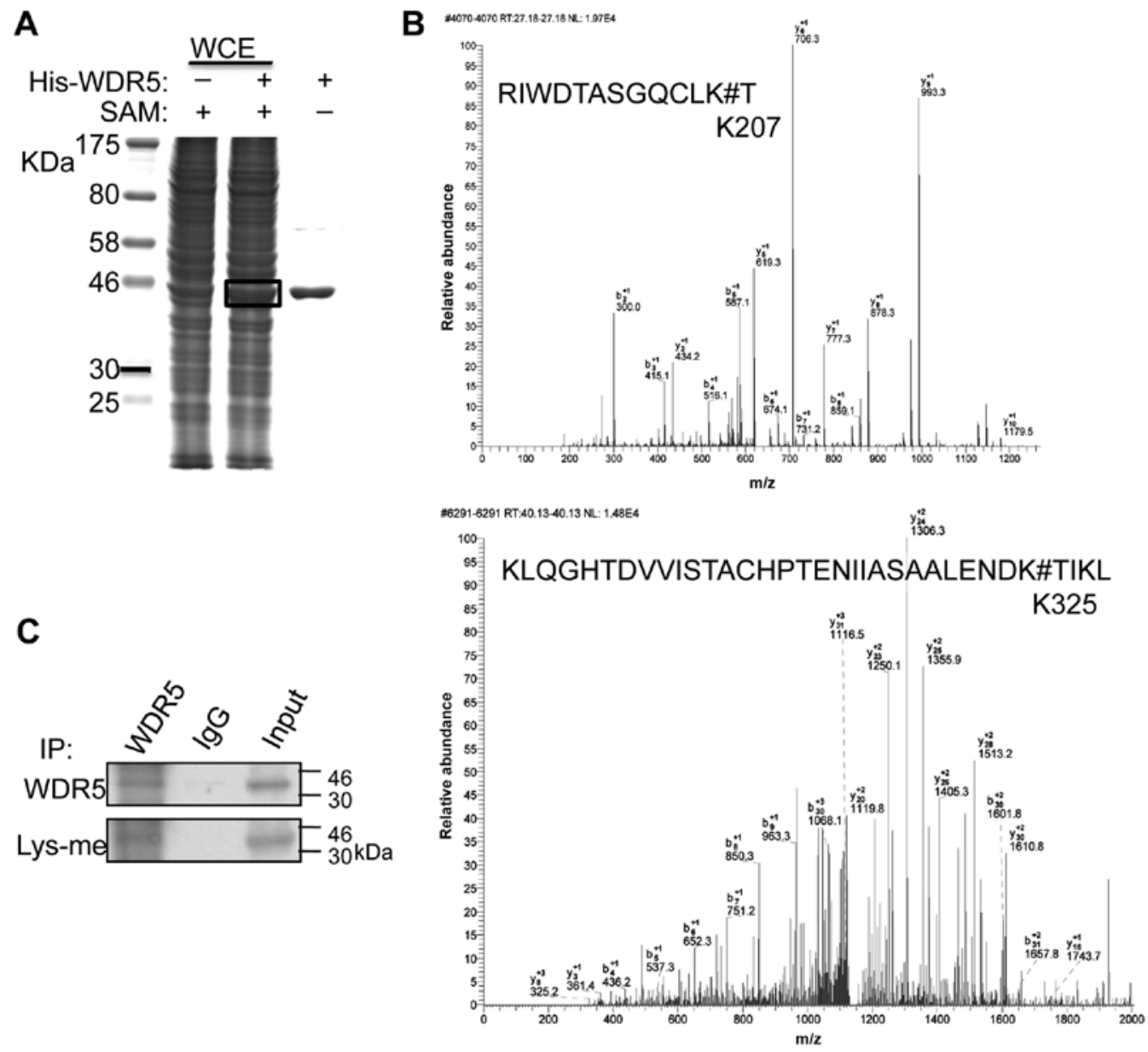

Figure 2. WDR5 was monomethylated at K207 and K325 in breast cancer cells. K207 and K325 have been identified as specific residues for WDR5 methylation by mass spectrometry analysis. (A) Using unlabeled SAM as a methyl donor, in vitro methylation reactions were performed and separated by SDS-PAGE. The band of His-WDR5 proteins (in the black box) stained by coomassie brilliant blue was excised for mass spectrometry. (B) Two monomethylated residues (K207 and K325) of WDR5 were identified from two WDR5 peptides. (C) Lysines of WDR5 were methylated in MDA-MB-231 cells. WDR5 proteins co-immunoprecipitated from the WCE of MDA-MB-231 cells by anti-WDR5 antibody were immunoblotted with Lys-me antibody. IgG was used as the negative control. WDR5, WD repeat domain 5; SAM, S-adenosyl methionine; His-, histidine-tagged; Lys-me, anti-methylated Lysine; IgG, immunoglobulin G; WCE, whole-cell extract.

$37^{\circ} \mathrm{C}$ with $5 \% \mathrm{CO}_{2}$ for two weeks. Fresh medium was changed every three days. At the end, the colonies were washed using PBS twice, fixed with methanol for $20 \mathrm{~min}$ at $-20^{\circ} \mathrm{C}$ and stained with $0.1 \%$ crystal violet for $10 \mathrm{~min}$ at room temperature.

Wound healing and cell migration assay. As described above, the Pwpxld plasmids expressing WT WDR5 and 2KR WDR5 were transfected into $293 \mathrm{~T}$ cells using PolyJet in vitro DNA transfection reagent (SignaGen Laboratories) for $6 \mathrm{~h}$ at room temperature. Lentiviral particles were incubated with MCF7 cells for $24 \mathrm{~h}$ to elevate WDR5 expression. Wound healing and cell migration assays were performed as previously described (24). For the wound healing assay, $3 \times 10^{5}$ MCF7 cells were seeded in 6 -well plates. The scratches were created using a $10 \mu \mathrm{l}$ pipette tip. After $24 \mathrm{~h}$, gap areas were then examined and photographed by Nomarski contrast microscope at a magnification of $\mathrm{x} 40$.

Statistical analysis. Statistical analysis was performed using GraphPad Prism 5 software (GraphPad Software, Inc., La Jolla, CA, USA). A unpaired Student's t-test was performed for the comparison of two groups and one-way analysis of variance followed by Newman-Keuls post hoc analysis was used for multiple group comparison. Error bars represented the mean \pm standard deviation of three independent replicates. $\mathrm{P}<0.05$ was considered to indicate a statistically significant difference.

\section{Results}

WDR5 is monomethylated at K207 and K325 in breast cancer cells. To explore if WDR5 was methylated in breast cancer cells, His-WDR5 proteins expressed in bacteria were purified (Fig. 1A). In vitro methylation assays were performed by incubating the purified proteins with whole-cell extracts of MDA-MB-231 breast cancer cells. MDA-MB-231 cells were used as a higher expression ofWDR5 was observed in these cells compared within mammary MCF10A epithelial cells and the breast cancer cell lines MCF7 and BT549 (Fig. 1B). Using ${ }^{3} \mathrm{H}-\mathrm{SAM}$ as a methyl donor, autoradiography revealed that His-WDR5 was methylated in vitro by whole-cell extracts of MDA-MB-231 cells (Fig. 1C).

To identify the specific residues of WDR5 methylated in MDA-MB-231 cells, an unlabeled SAM was used as a methyl donor for in vitro methylation assays as described. The band corresponding to His-WDR5 was excised for mass spectrometry analysis (Fig. 2A). A 14-Da shift was induced by addition 
of a single methyl group at K207 and K325 in two different WDR5 peptides, indicating these two lysine residues were monomethylated (Fig. 2B). Methylation of arginines was not detected in this analysis.

Immunoblotting with anti-methylated lysine antibody revealed the lysine methylation of WDR5 protein immunoprecipitated from whole-cell extracts of MDA-MB-231 cells (Fig. 2C). These data indicated that K207 and K325 of WDR5 are monomethylated in breast cancer cells.

K207 and K325 are key residues for the methylation of WDR5. To determine if K207 and/or K325 is preferentially methylated in breast cancer cells, each or both of these sites were mutated to arginine on His-WDR5 proteins expressed in bacteria. The proteins extracted were used for in vitro methylation assays. Methylation reactions without whole-cell extracts of MDA-MB-231 cells were used as negative controls. Immunoblotting with Lys-me antibody revealed that the methylation of WDR5 lysines decreased with K207R and K325R single-site mutations, and was eliminated by $2 \mathrm{KR}$ double-site mutation (Fig. 3A).

Once stable cell lines were established via virus infection, wild-type (WT) or mutated (K207R, K325R or K207R/K325R) Flag-WDR5 proteins were expressed at equal levels in 293T cells (Fig. 3B). WT or mutated Flag-WDR5 proteins were immunoprecipitated using anti-Flag M2 affinity gel, followed by immunoblotting with Lys-me antibody. A 2KR mutation disrupted the lysine methylation on WDR5 expressed in 293T cells (Fig. 3C). These data suggested that K207 and K325 were critical residues for WDR5 methylation by HMTs.

Methylation of K207/K325 promotes WDR5 in the enhancement of breast cancer cell proliferation and migration. WDR5 is reported to promote the proliferation of leukemia and bladder cancer cells $(8,9)$. To explore if WDR5 controlled the proliferation and migration of breast cancer cells, and if these functions of WDR5 were influenced by lysine methylation, WT or 2KR double-mutated WDR5 were overexpressed equally in MCF7 cells (data not shown), followed by a colony formation and cell migration assay.

The ectopic expression of WT WDR5 significantly increased the number of cell colonies formed $(\mathrm{P}<0.01$; Fig. 4A) and significantly enhanced cell migration $(\mathrm{P}<0.05$; Fig. 4B and C) compared with the vector. Increases in the percentage of cell colonies and migrating cell numbers induced by the overexpression of WT WDR5 were significantly attenuated by a $2 \mathrm{KR}$ double-mutation $(\mathrm{P}<0.05$; Fig. 4A-E). These results implied that the methylation of K207/K325 was crucial for WDR5 functions in promoting breast cancer cell proliferation and migration.

K207/K325 of WDR5 is methylated by SETD6 in vitro. To investigate whether HMTs that catalyze the methylation of K207/K325, GST-tagged HMTs, including SETD6, SET7/9, SET8 and SMYD3, were expressed and purified in bacteria. In vitro methylation reactions and ${ }^{3} \mathrm{H}$-labeling assays revealed that His-WDR5 lysines were methylated by GST-SETD6, but not GST-SET7/9, GST-SET8 or GST-SMYD3 (Fig. 5A and B).
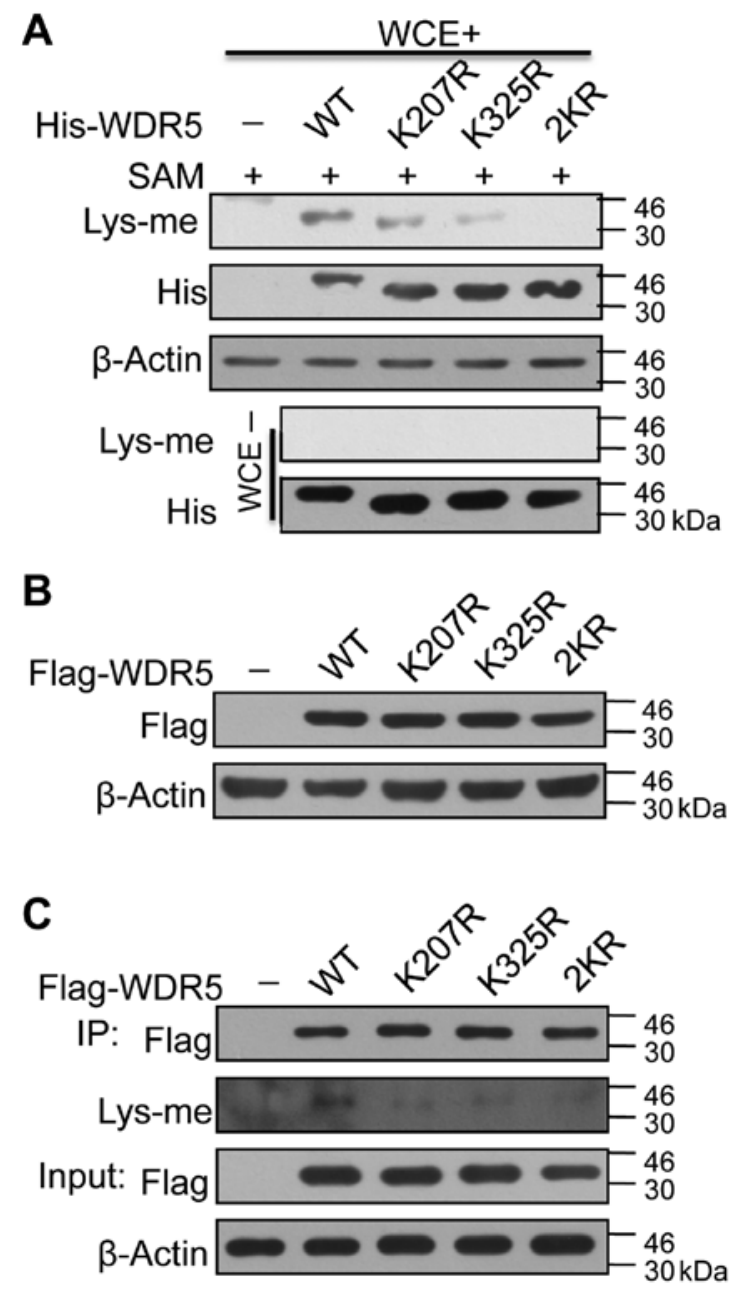

Figure 3. Lysine methylation of WDR5 decreased with K207R and K325R single-site mutation and was eliminated by $2 \mathrm{KR}$ double-site mutation. (A) Lysine methylation on WT or mutated His-WDR5 proteins were detected by immunoblotting in vitro methylation reactions with Lys-me antibody. Purified WT or mutated (K207R, K325R or 2KR) His-WDR5 proteins expressed in bacteria were incubated with $\left(\mathrm{WCE}^{+}\right)$or without $\left(\mathrm{WCE}^{-}\right)$the WCE of MDA-MB-231 cells for in vitro methylation assays. SAM was utilized as a methyl donor. $\beta$-Actin and His were used as internal references. K207R and K325R mean the mutations of lysine 207 and 325 of WDR5 into arginine. 2KR means the K207R/K325R double-site mutation of WDR5. (B) WT or mutated (K207R, K325R or 2KR) Flag-WDR5 were expressed at equal levels in $293 \mathrm{~T}$ cells by establishing stable cell lines via virus infection. p3*Flag-CMV-9/10 empty vector was used as the control vector. (C) Lysine methylation on WT or mutated Flag-WDR5 proteins co-immunoprecipitated from the WCE of $293 \mathrm{~T}$ cells were measured using a Lys-me antibody. $\beta$-Actin and Flag were used as the internal references. WDR5, WD repeat domain 5; WCE, whole-cell extract; WT, wild-type; 2KR, K207R/K325R double-site mutation; SAM, S-adenosyl methionine; Lys-me, anti-methylated Lysine; His-, histidine-tagged.

According to previous study, the HMT enzymatic activity of SETD6 is disrupted by a Y261A single-site mutation (25). This GST-SETD6 mutation was generated in the present study, and it was revealed that the lysine methylation on His-WDR5 was decreased by the Y261A mutation of GST-SETD6 and 2KR double-mutation of WDR5 (Fig. 5C). These results demonstrated that SETD6 was responsible for the methylation of K207/K325 on WDR5.

Methylation of K207/K325 is partially required for WDR5 in histone H3K4me3 generation. WDR5 is known to bind 
A

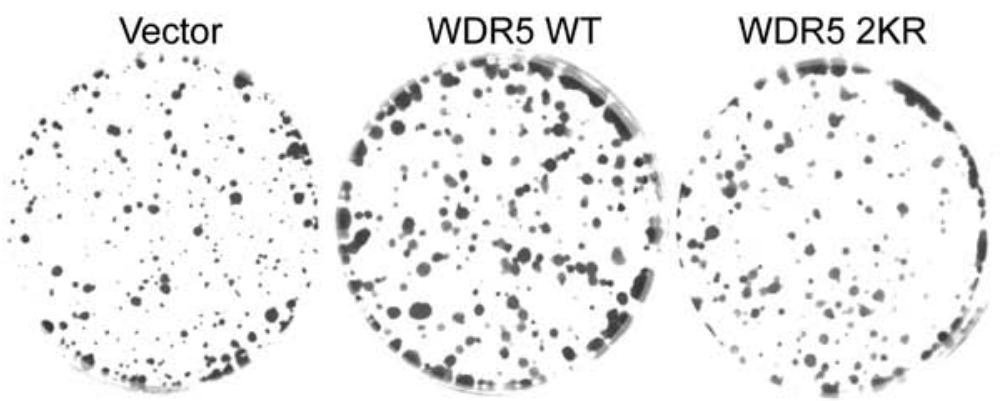

B
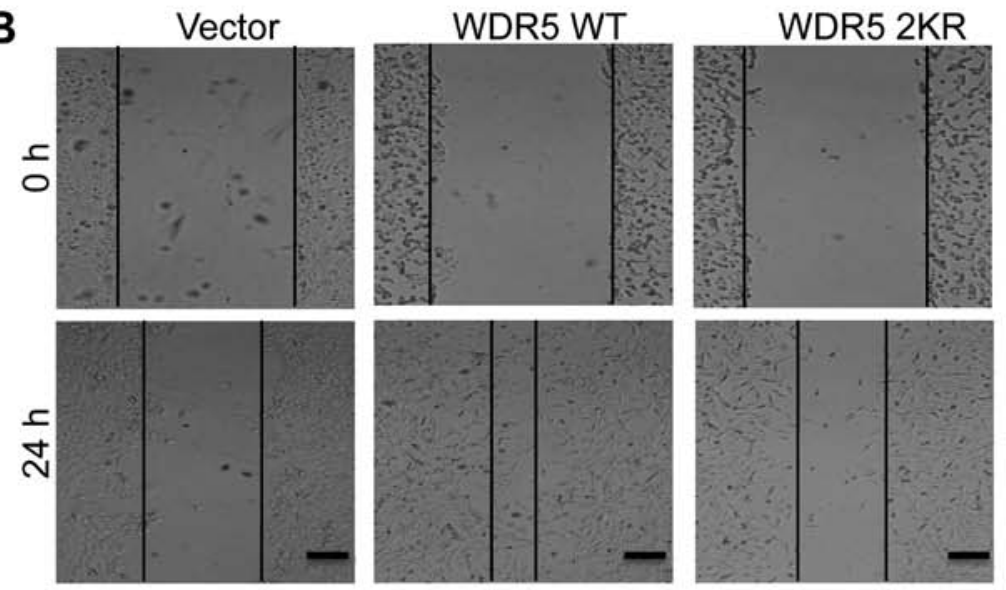

C
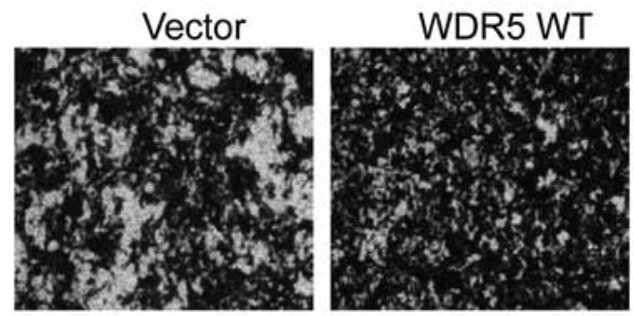

WDR5 2KR

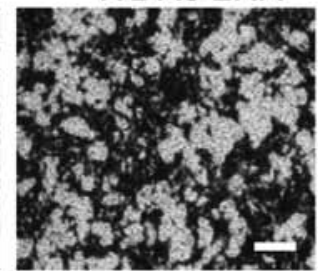

D

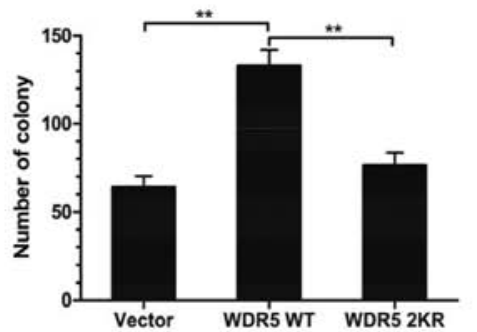

E

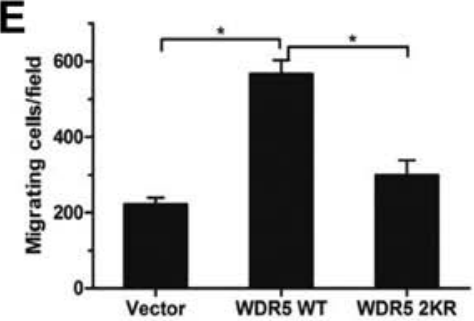

Figure 4. 2KR attenuated WDR5 functions in promoting cancer cell proliferation and migration. WT or mutated (K207R, K325R and 2KR) WDR5 proteins were expressed in MCF7 cells following virus infection. Representative images of (A) colony formation, (B) wound healing and (C) migration assays. Scale bar, $200 \mu \mathrm{m}$. (D) Number of colony and (E) percentage of migrating cells in identical fields were quantitatively analyzed. ${ }^{* *} \mathrm{P}<0.01$ and ${ }^{*} \mathrm{P}<0.05$ with comparisons shown by lines. 2KR, K207R/K325R double-site mutation; WDR5, WD repeat domain 5; WT, wild-type.

to histone H3K4me3 methyltransferase MLL1 to generate histone H3K4me3 (26). To investigate if the methylation of K207/K325 affected the interaction of WDR5 with MLL1, WT or 2KR double-mutated Flag-WDR5 were co-overexpressed with the His-tagged C-terminal SET domain of MLL1 in 293T cells. Co-immunoprecipitation experiments revealed that the 2KR mutation did not influence the binding of WDR5 to the C-terminal SET domain of MLL1 (Fig. 6A).

The global histone H3K4me3 levels were significantly decreased by the knockdown of WDR5 compared with the control $(\mathrm{P}<0.05$; Fig. 6B), verifying that WDR5 was responsible for histone $\mathrm{H} 3 \mathrm{~K} 4 \mathrm{me} 3$ generation. To investigate if histone $\mathrm{H} 3 \mathrm{~K} 4 \mathrm{me} 3$ generation altered with the methylation of K207/K325 on WDR5, WT and 2KR rWDR5 expression vectors were generated that were resistant to shWDR5\#1 due to asynonymous mutation (Fig. 6C). By the co-transfection of WT or mutated rWDR5 into 293T cells with shWDR5\#1, it was revealed that the overexpression of WT rWDR5 significantly restored the reduction in global histone H3K4me3 induced by shWDR5\#1 ( $\mathrm{P}<0.05$; Fig. 6D). This rescue was attenuated by disruption of K207/K325 methylation via a $2 \mathrm{KR}$ mutation (Fig. 6D). These results suggested that the methylation of K207/K325 was crucial for WDR 5 function in histone $\mathrm{H} 3 \mathrm{~K} 4 \mathrm{me} 3$ generation, although the methylation has no effect on the interaction of WDR5 with MLL1. 

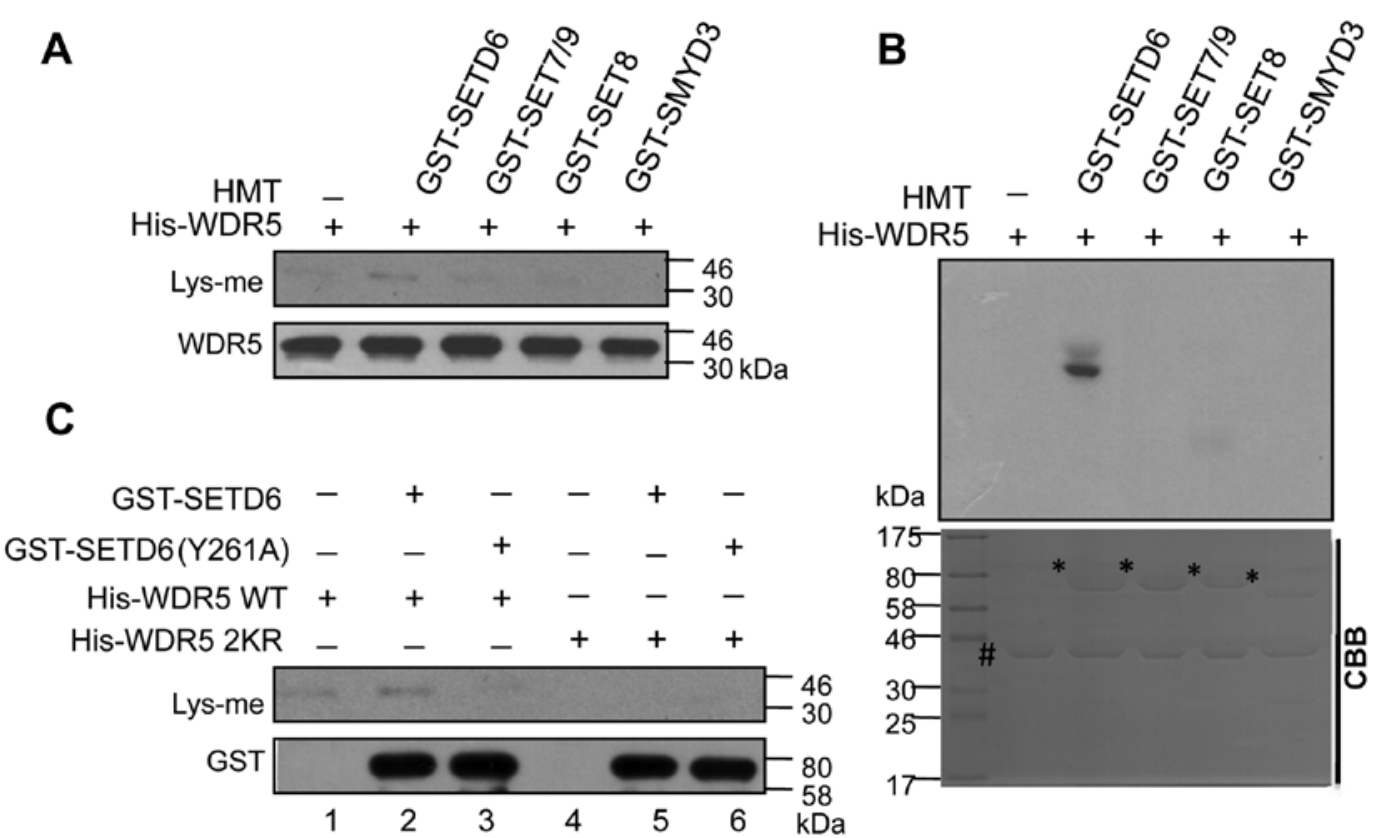

Figure 5. WDR5 was methylated by SETD6 in vitro. Purified His-WDR5 proteins expressed in bacteria were methylated by GST-SETD6 in vitro. His-WDR5 proteins were expressed and purified from bacteria, and incubated with GST-SETD6, GST-SET7/9, GST-SET8 or GST-SMYD3 for in vitro methylation assays. Lysine methylation on His-WDR5 proteins were detected by (A) immunoblotting with Lys-me antibody or (B) autoradiography assays. In (A), unlabeled $\mathrm{S}$-adenosyl methionine was used as a methyl donor, and WDR5 was used as an internal control for western blot analysis. In (B), S-adenosyl-L-[methyl- $\left.{ }^{-3} \mathrm{H}\right]$ methionine was used as a methyl donor, and CBB staining (lower) was used as a loading control for the autoradiography assays (upper). ${ }^{\#}$ Indicates the positions of His-WDR5 proteins, and * indicates the positions of GST-tagged proteins. (C) Lysine methylation on His-WDR5 proteins were diminished by a Y261A mutation of GST-SETD6 and 2KR mutation of His-WDR5. Purified WT or 2KR mutated His-WDR5 proteins expressed in bacteria were incubated with WT or Y261 A mutated GST-SETD6 for in vitro methylation reactions. Lysine methylation on His-WDR5 were detected by immunoblotting with a Lys-me antibody. GST was used as an internal control. WDR5, WD repeat domain 5; SETD6, SET domain containing protein methyltransferase 6; SET7/9, SET domain containing lysine methyltransferase 7/9; SET8, SET domain containing lysine methyltransferase 8; SMYD3, SET and MYND Domain Containing 3; His-, histidine-tagged; GST, Glutathione S-transferase; HMT, histone lysine methyltransferases; Lys-me, anti-methylated Lysine; WT, wild-type; 2KR, K207R/ K325R double-site mutation; CBB Coomassie brilliant blue.

\section{Discussion}

As a component of MLL/SET1, WDR5 is reported to promote cancer cell proliferation $(8,9)$. Whether WDR5 regulates cancer progression remains poorly understood. The present study reported that WDR5 expression levels increased in MDA-MB-231 highly metastatic breast cancer cells, compared with MCF7 and BT549 cells with low metastatic ability. Both the proliferation and migratory ability of MCF7 cells were elevated by the overexpression of WT WDR5, and these increases were attenuated by the disruption of WDR5 K207/K325 methylation. These results imply that the methylation of K207/K325 is vital for WDR5 promotion of breast cancer cell metastasis. Therefore, whether WDR5K207/K325 methylation is increased in mammary carcinomas tissue from patients with a poor clinical outcome requires further investigation.

The in vitro methylation assays in the present study revealed that WDR5 is methylated by SETD6. SETD6 contributes to the monomethylation of histone $\mathrm{H} 2 \mathrm{~A}$ variant $\mathrm{H} 2 \mathrm{AZ}$ on lysines 4 and 7 (27). Similar to WDR5, SETD6 is essential for the maintenance of self-renewal in embryonic stem cells (27) and is highly expressed in bladder cancer cells (9). Transcription factor nuclear factor (NF)-кB-p65 is monomethylated by SETD6, which increases the survival and colony formation of bladder cancer cells (28). Proteome-wide methodology has revealed that Polo-like Kinase 1 and p21-activated kinase 4 are methylated by SETD6 (29). The present study revealed that the 2KR double-mutation disrupted the WDR 5 methylation by SETD6, suggesting that SETD6 was responsible for catalyzing K207/K325 methylation. Whether SETD6 affects the function of WDR5 in promoting breast cancer cell progression is worth investigating in depth.

Crystal structure analysis reveals that the interactions between WDR5 and MLL is distinct from the interaction between WDR5 and histone H3, although WDR5 uses the same pocket to bind these two proteins (30). Glutamic acid 322 (E322) of WDR5, which is the key residue in WDR5-H3 complex structure, is not involved in the interaction of WDR5 with MLL $(2,30)$. The present study revealed that the methylation of K207/K325 on WDR5 is critical for maintaining global histone H3K4me3 levels, however, it does not affect MLL/SET1 complex assembly. As K207/K325 are close to E322, it was hypothesized that the methylation of K207/K325 enhances the structure of the WDR5-H3 complex to promote histone $\mathrm{H} 3 \mathrm{~K} 4 \mathrm{me} 3$ generation.

Protein methylation occurs at lysines and arginines. WDR5, absent, small, homeotic discs 2-like protein (Ash2L) and retinoblastoma-binding protein 5 form complexes with MLL/SET1 (31). Ash2L is asymmetrically dimethylated at arginine 296 by PRMT1, however, neither MLL complex integrity nor global histone $\mathrm{H} 3 \mathrm{~K} 4 \mathrm{me} 3$ levels are altered by this modification (32). However, no arginines on WDR5 were detected as methylated by the whole-cell extracts of breast 
A

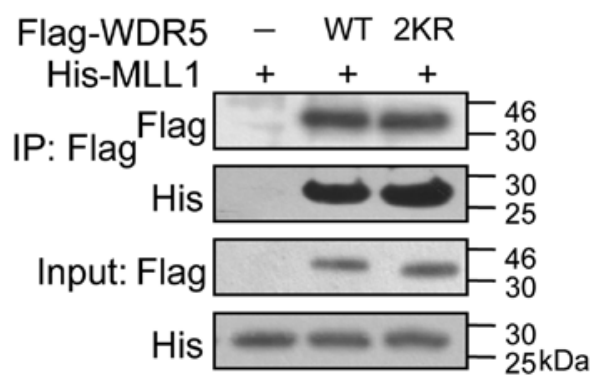

C Resistant WDR5 form (rWDR5)

\author{
shWDR5\#1 interference sequence: \\ GCAAGTTCATCTGCTGATA
}

\author{
GCAAGTTCATCTGCTGATAAA \\ $\downarrow$ correspond with \\ amino acids \\ ASSSADK \\ $\downarrow$ synonymous codons
}

GCCTCCAGCAGCGCGGACAAG
B

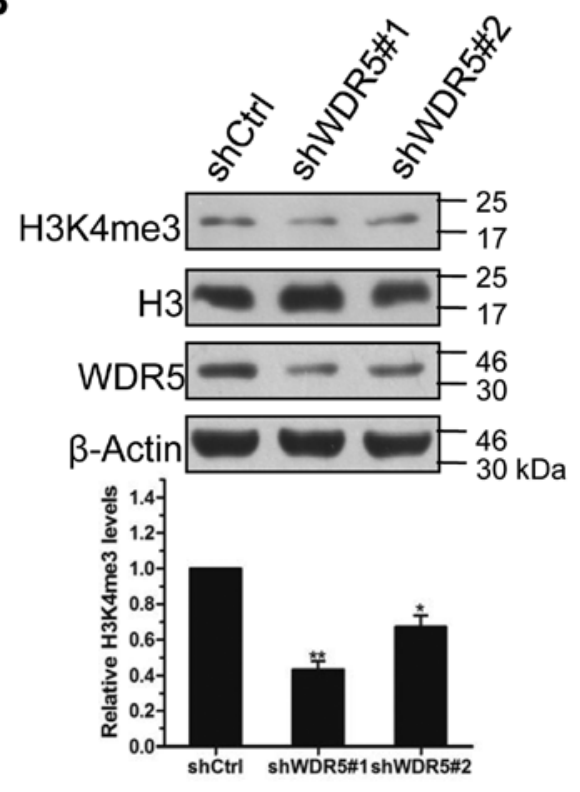

D

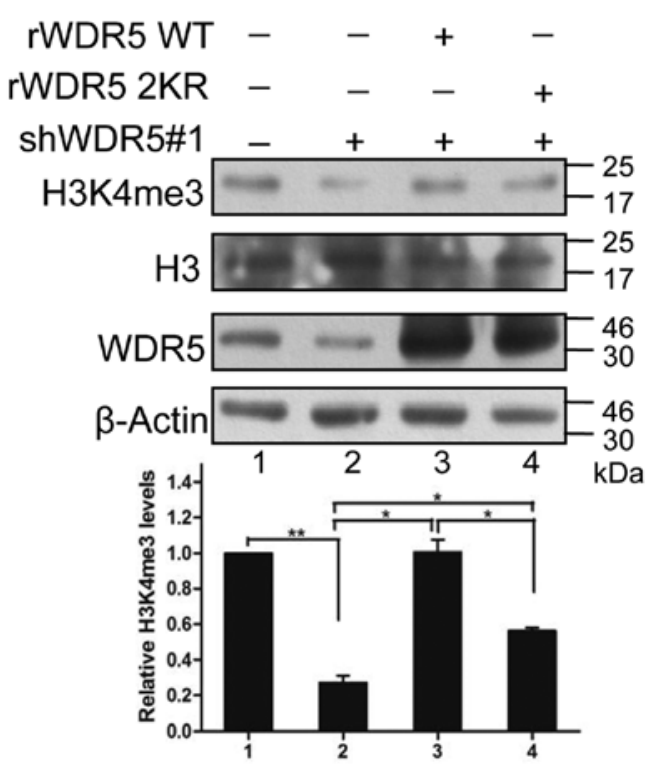

Figure 6. Methylation of K207/K325 is required for WDR5 in maintaining global histone H3K4me3 levels. (A) A 2KR did not affect the binding of WDR5 to MLL1. His-tagged C-terminal SET domain of MLL1 and WT or 2KR mutated Flag-WDR5 proteins were co-expressed in 293T cells. Flag-WDR5 proteins immunoprecipitated by Flag-conjugated agarose were immunoblotted with anti-Flag and anti-His antibodies. (B) Knockdown of WDR5 decreased global histone H3K4me3 levels. WDR5 expression was knocked down in 293T cells by specific shRNAs (shWDR5\#1 or shWDR5\#2). $\beta$-Actin was utilized as an internal reference. Global histone $\mathrm{H} 3 \mathrm{~K} 4 \mathrm{me} 3$ levels were detected by western blot analysis and quantitatively evaluated by densitometry. H3 was utilized as an internal reference. ${ }^{* *} \mathrm{P}<0.01$ and ${ }^{*} \mathrm{P}<0.05$ vs. shCtrl. (C) The flow path of generating rWDR5 by synonymous mutation. (D) K207R/K325R double-site mutation attenuated rWDR5 function in restoring the shWDR5\#1-induced reduction in global histone H3K4me3 levels. WT or 2KR mutated rWDR5 expression plasmids, and shWDR5\#1 plasmids were simultaneously expressed in 293T cells. Global histone H3K4me3 levels were detected by western blot analysis and quantitatively evaluated by densitometry analysis. H3 was utilized as an internal reference. ${ }^{* *} \mathrm{P}<0.01$ and ${ }^{*} \mathrm{P}<0.05$ with comparisons shown by lines. WDR5, WD repeat domain 5; His-, histidine-tagged; MLL1, mixed lineage leukemia; sh-, short hairpin RNA; H3K4me3, tri-methylation of lysine 4 on histone H3; H3, histone H3; WT, wild-type; 2KR, K207R/K325R double-site mutation; rWDR5, shWDR5\#1-resistant WDR5 gene; shCtrl, short hairpin RNA control.

cancer cells in mass spectrometry analysis. These results imply that WDR5 interacts with PRMTs indirectly.

WDR5 was identified as a substrate of SETD6 and it was revealed that the methylation of specific lysines (K207/K325) of WDR 5 was critical for maintaining global histone H3K4me3 levels and promoting breast cancer cell proliferation and migration. These results imply the potential of exploring chemicals to inhibit K207/K325 methylation as an antitumor therapeutic target.

\section{Acknowledgements}

Not applicable.

\section{Funding}

The present study was supported by the National Natural Science Foundation of China (grant nos. 31000575 to Dr Xiaoxue Li, 31571317 to Dr Jun Lu, 31401105 to Dr Jiang Tan, and 81600173 to Dr Ruosi Yao), the Postdoctoral Science Foundation of China (grant no. 2016M601895), the Jilin Scientific and Technological Program (grant nos. 20150101069JC to Dr Xiaoxue Li, 20160101157JC to Dr Guiying $\mathrm{Xu}$, and 20140520003JH to Dr Jiang Tan), the Natural Science Foundation of Jiangsu Province (grant no. BK20160230), and the Postdoctoral Science Foundation of Jiangsu Province (grant no. 1601092B). 


\section{Availability of data and materials}

The datasets used and/or analyzed during the current study are available from the corresponding author on reasonable request.

\section{Authors' contributions}

RY and YW conceived and designed the experiments. RY, DH and YM performed the experiments. MM, YZ and JT prepared the solutions and cultured cells. JL and GX analyzed the data. RY and XL wrote the paper. All authors read and approved the final manuscript.

\section{Ethics approval and consent to participate}

The present study does not contain any studies involving human participants or animals that were performed by any of the authors.

\section{Patient consent for publication}

Not applicable.

\section{Competing interests}

The authors declare that they have no competing interests.

\section{References}

1. Dou Y, Milne TA, Ruthenburg AJ, Lee S, Lee JW, Verdine GL, Allis CD and Roeder RG: Regulation of MLL1 H3K4 methyltransferase activity by its core components. Nat Struct Mol Biol 13: 713-719, 2006.

2. Han Z, Guo L, Wang H, Shen Y, Deng XW and Chai J: Structural basis for the specific recognition of methylated histone $\mathrm{H} 3$ lysine 4 by the WD-40 protein WDR5. Mol Cell 22: 137-144, 2006.

3. Ang YS, Tsai SY, Lee DF, Monk J, Su J, Ratnakumar K, Ding J, Ge Y, Darr H, Chang B, et al: Wdr5 mediates self-renewal and reprogramming via the embryonic stem cell core transcriptional network. Cell 145: 183-197, 2011.

4. Wysocka J, Swigut T, Milne TA, Dou Y,Zhang X, Burlingame AL, Roeder RG, Brivanlou AH and Allis CD: WDR5 associates with histone $\mathrm{H} 3$ methylated at $\mathrm{K} 4$ and is essential for $\mathrm{H} 3 \mathrm{~K} 4$ methylation and vertebrate development. Cell 121: 859-872, 2005.

5. Kim JY,Banerjee T, Vinckevicius A, Luo Q, Parker JB, Baker MR, Radhakrishnan I, Wei JJ, Barish GD and Chakravarti D: A role for WDR5 in integrating threonine 11 phosphorylation to lysine 4 methylation on histone $\mathrm{H} 3$ during androgen signaling and in prostate cancer. Mol Cell 54: 613-625, 2014.

6. Chen X, Gu P, Li K, Xie W, Chen C, Lin T and Huang J: Gene expression profiling of WDR5 regulated genes in bladder cancer. Genom Data 5: 27-29, 2015.

7. Tan X, Chen S, Wu J, Lin J, Pan C, Ying X, Pan Z, Qiu L, Liu R, Geng R, et al: PI3K/AKT-mediated upregulation of WDR5 promotes colorectal cancer metastasis by directly targeting ZNF407. Cell Death Dis 8: e2686, 2017.

8. Ge Z, Song EJ, Kawasawa YI, Li J, Dovat S and Song C: WDR5 high expression and its effect on tumorigenesis in leukemia. Oncotarget 7: 37740-37754, 2016.

9. Chen X, Xie W, Gu P, Cai Q, Wang B, Xie Y, Dong W, He W, Zhong G, Lin T, et al: Upregulated WDR5 promotes proliferation, self-renewal and chemoresistance in bladder cancer via mediating H3K4 trimethylation. Sci Rep 5: 8293, 2015.

10. Clarke SG: Protein methylation at the surface and buried deep: Thinking outside the histone box. Trends Biochem Sci 38: 243-252, 2013

11. Shi Y and Whetstine JR: Dynamic regulation of histone lysine methylation by demethylases. Mol Cell 25: 1-14, 2007.
12. Bedford MT and Clarke SG: Protein arginine methylation in mammals: Who, what, and why. Mol Cell 33: 1-13, 2009.

13. Chuikov S, Kurash JK, Wilson JR, Xiao B, Justin N, Ivanov GS, McKinney K, Tempst P, Prives C, Gamblin SJ, et al: Regulation of p53 activity through lysine methylation. Nature 432: 353-360, 2004.

14. Huang J, Perez-Burgos L, Placek BJ, Sengupta R, Richter M, Dorsey JA, Kubicek S, Opravil S, Jenuwein T and Berger SL: Repression of p53 activity by Smyd2-mediated methylation. Nature 444: 629-632, 2006.

15. Shi X, Kachirskaia I, Yamaguchi H, West LE, Wen H, Wang EW, Dutta S, Appella E and Gozani O: Modulation of p53 function by SET8-mediated methylation at lysine 382. Mol Cell 27: 636-646, 2007.

16. Vershinin Z, Feldman M, Chen A and Levy D: PAK4 methylation by SETD6 promotes the activation of the Wnt/ $\beta$-catenin pathway. J Biol Chem 291: 6786-6795, 2016.

17. Bedford MT and Richard S: Arginine methylation an emerging regulator of protein function. Mol Cell 18: 263-272, 2005.

18. Wang L, Zhao Z, Meyer MB, Saha S, Yu M, Guo A, Wisinski KB, Huang W, Cai W, Pike JW, et al: CARM1 methylates chromatin remodeling factor BAF155 to enhance tumor progression and metastasis. Cancer Cell 25: 21-36, 2014

19. Jansson M, Durant ST, Cho EC, Sheahan S, Edelmann M, Kessler B and La Thangue NB: Arginine methylation regulates the p53 response. Nat Cell Biol 10: 1431-1439, 2008.

20. Geng P, Zhang Y, Liu X, Zhang N, Liu Y, Liu X, Lin C, Yan X, Li Z, Wang G, et al: Automethylation of protein arginine methyltransferase 7 and its impact on breast cancer progression. FASEB J 31: 2287-2300, 2017.

21. Yao R, Jiang H, Ma Y, Wang L, Wang L, Du J, Hou P, Gao Y, Zhao L, Wang G, et al: PRMT7 induces epithelial-to-mesenchymal transition and promotes metastasis in breast cancer. Cancer Res 74: 5656-5667, 2014.

22. Zhao L, Zhang Y, Gao Y, Geng P, Lu Y, Liu X, Yao R, Hou P, Liu D, Lu J, et al: JMJD3 promotes SAHF formation in senescent WI38 cells by triggering an interplay between demethylation and phosphorylation of RB protein. Cell Death Differ 22: 1630-1640, 2015.

23. Zhao D and Huang Z: Effect of His-tag on expression, purification, and structure of zinc finger protein, ZNF191 (243-368). Bioinorg Chem Appl 2016: 8206854, 2016.

24. Hou P, Zhao Y, Li Z, Yao R, Ma M, Gao Y, Zhao L, Zhang Y, Huang $B$ and Lu J: LincRNA-ROR induces epithelial-to-mesenchymal transition and contributes to breast cancer tumorigenesis and metastasis. Cell Death Dis 5: e1287, 2014.

25. Levy D, Kuo AJ, Chang Y, Schaefer U, Kitson C, Cheung P, Espejo A, Zee BM, Liu CL, Tangsombatvisit S, et al: Lysine methylation of the NF- $\mathrm{kB}$ subunit RelA by SETD6 couples activity of the histone methyltransferase GLP at chromatin to tonic repression of NF- $\mathrm{BB}$ signaling. Nat Immunol 12: 29-36, 2011.

26. Trievel RC and Shilatifard A: WDR5, a complexed protein. Nat Struct Mol Biol 16: 678-680, 2009.

27. Binda O, Sevilla A, LeRoy G, Lemischka IR, Garcia BA and Richard S: SETD6 monomethylates H2AZ on lysine 7 and is required for the maintenance of embryonic stem cell self-renewal. Epigenetics 8: 177-183, 2013.

28. Mukherjee N, Cardenas E, Bedolla R and Ghosh R: SETD6 regulates NF- $\kappa \mathrm{B}$ signaling in urothelial cell survival: Implications for bladder cancer. Oncotarget 8: 15114-15125, 2017.

29. Levy D, Liu CL, Yang Z, Newman AM, Alizadeh AA, Utz and Gozani O: A proteomic approach for the identification of novel lysine methyltransferase substrates. Epigenetics Chromatin 4: 19, 2011.

30. Song JJ and Kingston RE: WDR5 interacts with mixed lineage leukemia (MLL) protein via the histone H3-binding pocket. J Biol Chem 283: 35258-35264, 2008.

31. Dharmarajan V, Lee JH, Patel A, Skalnik DG and Cosgrove MS: Structural basis for WDR5 interaction (Win) motif recognition in human SET1 family histone methyltransferases. J Biol Chem 287: 27275-27289, 2012.

32. Butler JS, Zurita-Lopez CI, Clarke SG, Bedford MT and Dent SY: Protein-arginine methyltransferase 1 (PRMT1) methylates Ash2L, a shared component of mammalian histone H3K4 methyltransferase complexes. J Biol Chem 286: 12234-12244, 2011. 